ISSN 1112-9867

Available online at http://www.jfas.info

\title{
DAIRY PRODUCTION AND SOURCES OF CELLULAR VARIABILITY OF MILK OF COW'S IN TWO LARGE FARMS IN SEMI-ARID ENVIRONMENT (TUNISIA COASTAL)
}

\author{
Y. M'Sadak* and I. Hamed
}

Institut Supérieur Agronomique de Chott-Mariem, Université de Sousse, Tunisie

Received: 19 October 2017 / Accepted: 16 April 2017 / Published online: 01 May 2017

\begin{abstract}
This investigation, referring to a description of the Individual Counts Cellular (CCI) of milk, accompanied by an analysis, so some quantitative and qualitative milk parameters that a number of physiological and zootechnical factors of cellular evolution, was performed on two large cattle farms conducted in semi-arid coastal environment (region of Sousse, Tunisia), subject tri-daily in mechanical milking room. It is especially striking that the arithmetic mean (MA) of the CCI cows's was 371000 cell. / ml, while the geometric mean (MG) of the CCI cows's was only 107500 cell. / ml. The median was 73,000 cells. / $\mathrm{ml}$, value close to the MG and widely spaced from the MA, thus telling the usefulness of the MG. A great heterogeneity of cell quality of the milk was noted, however, without clearly identify correlation with the chemical composition of milk. In two large herds followed, the variation of CCI was inversely related to dairy productions. On the other hand, the CCI found an increase with the rank of lactation, the stage of lactation and the spring season of calving.
\end{abstract}

Keywords: Dairy cattle, Cellular Counts Individual, milk production level, quality milk, Factors of cellular variation, semi-arid coastal environment.

Author Correspondence, e-mail: msadak.youssef@yahoo.fr

doi: http://dx.doi.org/10.4314/jfas. v9i2.16 


\section{INTRODUCTION}

Depuis 1990, la Tunisie a atteint l'autosuffisance en lait avec un accroissement continu de la production. Les aspects qualitatifs du lait à la production sont restés défaillants [1]. Il est souhaitable que le développement quantitatif constaté soit accompagné d'un développement qualitatif, afin de satisfaire les requêtes du consommateur au sujet de la valeur sanitaire du lait et celle du transformateur à propos de la composition chimique [2, 3].

Les mammites sont, depuis l'apparition de la traite mécanique, sources des pertes économiques les plus importantes en élevage bovin laitier [4] et peuvent représenter un danger pour la santé humaine, notamment avec les fromages au lait cru [5]. Les pertes correspondent au coût du traitement, aux réformes de vaches incurables et aux baisses de production laitière [3]. Le dénombrement des cellules somatiques, reflétant la santé de la glande mammaire [6], est utilisé comme caractère indicateur d'une infection mammaire clinique ou subclinique de la vache [7]. Un lait avec un dénombrement de cellules somatiques peu élevé est synonyme de vaches avec un pis en santé et de production d'un lait d'excellente qualité [8]. Lorsque le pis est exempt d'infection, il ne réplique pas et on se sert de cela comme d'un indice de qualité du lait [6]. Le suivi cellulaire mensuel admet d'avoir une idée exacte de la dynamique des infections mammaires [9].

Certains facteurs pourraient être à l'origine de l'évolution des Comptages Cellulaires Individuels (CCI). La race, le rang et le stade de lactation, le mois et la saison du vêlage, la production laitière individuelle, sont les sources essentielles pouvant influencer les CCI [10, 11, 12, 13, 14, 15, 16, 17, 18, 19]. C'est dans cette optique que les objectifs de cette étude étaient d'établir l'état des lieux des productions quantitative et qualitative du lait de vache et de ses variations cellulaires selon certains facteurs physiologiques et zootechniques courant la lactation. L'accent a été mis particulièrement sur les CCI en rapport avec les variables de production et de qualité laitières chez deux grands élevages bovins conduits en environnement semi-aride de la Tunisie.

\section{MATÉRIEL ET MÉTHODES}

\subsection{Contexte général de l'étude}

La présente investigation s'est intéressée à deux grands troupeaux bovins laitiers appartenant à deux zones voisines du gouvernorat de Sousse, région appartenant du Sahel Tunisien. La première est une exploitation privée de Mohamed Gloulou, située à Sidi Bou Ali (Ferme 1) et la deuxième est une exploitation étatique (Agro-combinat Enfidha) dépendant de l'Office des Terres Domaniales (Ferme 2). L'étude a été accomplie sur ces deux élevages inscrits au Contrôle Laitier (CL) rassemblant dans l'ensemble 185 Vaches Présentes (VP) et 154 Vaches en Lactation (VL), durant une période de suivi effectif, étalée de Novembre 2012 à Juin 2013. La répartition des 
deux élevages enquêtés est donnée dans le Tableau 1. La race discernée étant Frisonne Holstein. La traite étant tri-quotidienne (matin, après-midi et soir), en ayant recours au système en salle respectivement du type lactoduc en ligne haute simple rangée sans fosse (Ferme 1) et du type lactoduc en ligne intermédiaire double rangée en épi avec fosse (Ferme 2). Le nombre adopté de postes de traite est 10 pour la Ferme 1. La Ferme 2 dispose de 6 postes par quai (12 au total). Dans les deux exploitations, on a fait appel au double équipement caractérisé par un nombre des stalles égal au nombre de faisceaux-trayeurs.

Tableau 1. Distribution et caractéristiques des deux élevages bovins laitiers suivis

\begin{tabular}{cccccccccc}
\hline Ferme & Zone & Établissement & $\begin{array}{c}\text { Production } \\
\text { annuelle } \\
\end{array}$ & Contrôle & \multicolumn{2}{c}{ VP* } & \multicolumn{3}{c}{ VL* $^{*}$} \\
$\mathbf{1}$ & $\begin{array}{c}\text { Sidi Bou } \\
\text { Ali }\end{array}$ & Privé & 344672 & AT6** & 68 & 33,5 & 54 & 35 \\
$\mathbf{2}$ & Enfidha & Étatique & 748509 & B6*** & 117 & 66,5 & 100 & 65 \\
& & & & & 185 & 100 & 154 & 100 \\
\hline
\end{tabular}

*: Nombre moyen des vaches durant le suivi réalisé.

**: CL effectué par un Technicien de l'Office de l'Élevage et des Pâturages (OEP), toutes les six semaines.

*** CL réalisé par l'Éleveur (Technicien de la ferme), toutes les six semaines.

La Ferme 1 pratique le système d'élevage hors sol, déterminé par l'insuffisance des ressources fourragères, à cause des ressources hydriques généralement limitées tant quantitativement que qualitativement (milieu littoral semi-aride). La Ferme 2, disposant des ressources et des infrastructures hydrauliques souterraines, fait recours à l'agriculture irriguée, tout en adoptant ainsi le système d'élevage intensif intégré, caractérisé par une alimentation à base de la verdure et de l'ensilage.

Ces deux exploitations optent pour la stabulation libre (aire paillée) sans recourir à un système de drainage des effluents d'élevage générés. La fréquence, tant de paillage que de curage de l'étable, est quotidienne dans la Ferme 1, alors que dans la Ferme 2, le paillage est adopté un jour sur deux et le curage est saisonnier.

\subsection{Prélèvements du lait}

Les échantillons laitiers ont été prélevés en alternance pendant les trois horaires de traite (matin, après-midi et soir). Les deux installations de traite sont équipées d'un compteur à lait mécanique. Durant son utilisation, l'appareil doit être immobilisé verticalement. Pour éviter que les vaches puissent faire tomber l'appareil, il peut être maintenu au moyen d'une ficelle ou d'un crochet. Pour éviter la coagulation du lait depuis le moment du prélèvement de l'échantillon jusqu'à l'analyse au laboratoire, chaque flacon est pourvu du bichromate de potassium comme 
conservateur. Les échantillons ne doivent pas surpasser la limite de 10 jours de conservation à $4^{\circ} \mathrm{C}$. Les analyses du lait ont été exécutées au sein du Laboratoire du Service de Contrôle Laitier du Centre d'Amélioration Génétique de Sidi Thabet, à l'aide d'un compteur cellulaire automatique de type Fossomatic 4000, mis au point par la Société Danoise Foss Electric, indiquant les résultats cellulaires selon la méthode fluoro-opto-électronique qui constitue un moyen direct de dénombrement des cellules somatiques du lait [20].

\subsection{Données du contrôle laitier}

Les relevés bi-trimestriels (toutes les 6 semaines par un Technicien de l'OEP pour AT6 et par l'Éleveur pour B6) du CL des vaches laitières constituent la méthode de recueil de renseignements objectifs et ils sont disponibles en permanence à la Direction Régionale de l'OEP de Sousse pour les élevages adhérents au CL. Les fiches retenues datent du mois de Mai 2012 jusqu'au mois d'Avril 2013. Durant cette période, on a pu relever les données du suivi de 184 VL (Dépouillement de 3 à 8 contrôles disponibles par lactation considérée). Autrement dit, cette période est bornée par le premier vêlage et le dernier tarissement pris en compte.

\subsection{Analyse statistique descriptive des données du Contrôle Laitier}

Les données des CCI, les données de la Production Laitière (PL), ainsi que de la composition chimique du lait : Matière Grasse (MG), Matière Protéique (MP) et Urée, ont été relevées en ayant recours aux fiches disponibles dans la base nationale des données du CL de l'OEP.

Après dépouillement des CCI, ces derniers ont subi un traitement statistique par le logiciel SAS (version 9.13). On a calculé les moyennes arithmétiques (MA) et géométriques (MG) pour chaque contrôle (du premier au huitième contrôle) dont les formules de calcul sont :

$$
\mathbf{M A}=\sum_{1}^{\mathrm{n}} \mathrm{CCI} / \mathbf{N} \quad \text { et } \quad \mathbf{M G}=\sqrt[\mathrm{n}]{\operatorname{CCI} 1 \times \text { CCI } 2 \times \ldots \times \text { CCIn }}[14]
$$

Avec :

CCI : Comptage Cellulaire Individuel

$\mathrm{N}$ : Nombre total des échantillons de lait.

Les écarts-types, les fréquences, les quartiles (Q1, Q2 et Q3) et l'écart interquartile (Q3-Q1) des valeurs des CCI ont été également dénombrés pour mieux apprécier la répartition des données cellulaires.

Signalons que la répartition des CCI de 8 contrôles considérés a été déterminée pour chaque contrôle et pour chaque ferme en ayant recours aux paramètres statistiques suivants : Moyenne, Écart-type et Valeurs Extrêmes (Minimum et Maximum). Ensuite, pour chaque ferme, la distribution des $\mathrm{CCI}$ de 8 contrôles examinés $(\mathrm{C} 1$ à $\mathrm{C} 8)$ a été présentée, à titre comparatif, selon 
les deux méthodes de calcul de la moyenne, à savoir MA et MG. Ultérieurement, le diagramme des quartiles des CCI a été établi pour chaque ferme.

\subsection{Analyse des facteurs d'évolution de la qualité cellulaire du lait de vache}

Il s'agit d'étudier notamment les paramètres de variation suivants : production laitière individuelle, rang de lactation, stade de lactation, mois et saison de vêlage. De telles variables d'évolution des CCI ont été analysées pour chaque ferme.

\section{RÉSULTATS ET DISCUSSION}

\subsection{Caractérisations zootechnique et cellulaire}

\subsubsection{Quelques variables de production et de reproduction}

Les vaches en production (VL) de la Ferme 1, renfermant un effectif de 54, en moyenne, se trouvent groupées dans un même lot, alors qu'à la Ferme 2, les 100 VL existantes sont distribuées selon leur niveau de production en quatre groupes. Le premier groupe (Lot 1) est celui des hautes productrices (> 25 1), le deuxième renferme les vaches ayant une moyenne de production entre 20 et 251 , le troisième est celui des vaches produisant entre 15 et 201 , et le dernier groupe (Lot 4) est celui des vaches qui génèrent moins de 151 .

Le Tableau 2, à caractère synthétique, montre la répartition des vaches considérées selon leur production laitière (PL), le rang de lactation, la moyenne de lactation et la saison de vêlage. La moyenne de production laitière par vache en lactation (VL), pour les deux Fermes, est presque la même. On remarque, malgré la différence de conduite et d'effectif des primipares, la production laitière par vache est semblable. D'ailleurs pour la moyenne de lactation, on note la même marge par vache. On a observé que le cheptel de la Ferme 2 est, en grande partie, jeune avec $43 \%$ des vaches primipares. Cette catégorie des vaches présente strictement le tiers dans la Ferme 1. Concernant la saison de vêlage, la majorité des vêlages se concentre lors de la période froide (Automne + Hiver) avec un pourcentage moyen de $61 \%$ des mises bas.

Tableau 2. Répartition des vaches selon des grandeurs de production et de reproduction

\begin{tabular}{|c|c|c|c|c|c|c|c|c|}
\hline \multirow[t]{2}{*}{ Ferme } & \multirow{2}{*}{$\begin{array}{c}\text { Niveau de } \\
\text { production } \\
(1 / \mathrm{VL} / \mathrm{j})\end{array}$} & \multicolumn{2}{|c|}{ Rang de lactation (\%) } & \multirow{2}{*}{$\begin{array}{l}\text { Moyenne de } \\
\text { lactation }\end{array}$} & \multicolumn{4}{|c|}{$\begin{array}{c}\text { Saison de vêlage* } \\
(\%)\end{array}$} \\
\hline & & Primipares & Multipares & & $\mathbf{H}$ & $\mathbf{P}$ & $\mathbf{E}$ & $\mathbf{A}$ \\
\hline 1 & $19 \pm 4$ & 32 & 68 & $3 \pm 2$ & 37 & 22 & 23 & 18 \\
\hline 2 & $19 \pm 5$ & 43 & 57 & $3 \pm 2$ & 30 & 14 & 23 & 33 \\
\hline Moyenne & 19 & 39 & 61 & $3 \pm 2$ & 33 & 17 & 22 & 28 \\
\hline
\end{tabular}

*H: Hiver $;$ P : Printemps ; E : Eté ; A : Automne 


\subsubsection{Présentation des CCI}

Les comptages cellulaires individuels (CCI) souscrivent la gestion des situations d'urgence au cours de la lactation et l'assainissement du troupeau lors de la période sèche [3]. Le CCI est un repère de l'état inflammatoire de la mamelle et indirectement de l'existence d'une infection mammaire [13].

Les statistiques élémentaires (Moyennes, Écart-Type, Extrêmes) des CCI durant les huit contrôles réalisés sont relatées dans le Tableau 3. La moyenne arithmétique (MA) des CCI des vaches de la Ferme 1 a été de 386000 cell. /ml, et celle des vaches de la Ferme 2 a été de 355000 cell. $/ \mathrm{ml}$, soit une valeur moyenne de 371000 cell. $/ \mathrm{ml}$ au niveau des deux fermes. Cette moyenne est importante par rapport au seuil de 300000 cell. /ml fixé par Fabre et al [21] et trop éloignée du seuil de 200000 cell. /ml rapporté par Noireterre [3]. La moyenne géométrique (MG) des vaches des deux élevages a été seulement de 107500 cell. /ml, avec une MG de 108000 cell. /ml des vaches de la Ferme 1 et de 107000 cell. /ml des vaches de la Ferme 2. On constate, alors, que les CCI apparaissent plus élevés avec la méthode d'estimation basée sur la MA, comparativement avec celle basée sur la MG.

La condition sanitaire mammaire des deux élevages suivis est considérée meilleure par rapport à celle d'autres grands élevages suivis dans le passé dans le Nord Tunisien (berceau des grandes exploitations laitières), où la moyenne arithmétique du comptage cellulaire collectif était de 626000 cell. $/ \mathrm{ml}$ [22]. Certes, la situation n'est pas comparable en raison tant d'une nette amélioration, entre temps, des conditions d'élevage et de traite chez les grands troupeaux que des méthodes différentes de comptage cellulaire (d'une part, sur lait individuel, et d'autre part, sur lait de mélange). Également, si l'on compare à une autre étude plus récente dans une région voisine de Sousse, qui est le gouvernorat de Monastir, les moyennes arithmétiques et géométriques trouvés sont beaucoup plus meilleures. En effet, Mighri et al. [23] ont relevé une moyenne arithmétique des CCI des vaches, chez des petits élevages bovins hors sol, qui était de 640000 cell. /ml, alors que la moyenne géométrique (MG) était de 233000 cell. /ml. On constate, ainsi, qu'à l'échelle de la Tunisie, les résultats de CCI trouvés méritent d'être considérés admissibles lors de cette étude, soit par rapport aux autres élevages du Sahel Tunisien majoritairement familiaux, soit par rapport aux grands élevages du Nord Tunisien (contexte ancien à considérer avec prudence). 
Tableau 3. Analyse statistique élémentaire des CCI

\begin{tabular}{lcccc}
\hline & \multirow{2}{*}{ Variables } & \multicolumn{3}{c}{ CCI (x1000 cell./ml) } \\
\cline { 3 - 5 } & Ferme 1 & Ferme 2 & Échantillon total suivi \\
\hline MA $^{*}$ & 386 & 355 & 371 \\
MG $^{*}$ & 108 & 107 & 107,5 \\
E-T $^{*}$ & 1137 & 1069 & 1103 \\
Min. $^{*}$ & 3 & 4 & - \\
Max. $^{*}$ & 17232 & 29364 & - \\
\hline
\end{tabular}

(*) Moyennes arithmétique (MA) et géométrique $(M G)$; Écart-type (E-T) ; Extrêmes Minimum (Min.) et Maximum (Max.).

Pendant la période considérée de l'étude, on a enregistré un minimum de 1000 cell. $/ \mathrm{ml}$ dans la Ferme 2 et 3000 cell. /ml dans la Ferme 1, lors du dernier contrôle (mois d'Avril). Aussi, on a repéré un maximum de 29364000 cell. /ml, dans la Ferme 2, et 17232000 cell. /ml, à la Ferme 1, durant le troisième contrôle (mois de Juillet).

On a noté, autant, que l'écart des résultats est grand (Ecart-type trop élevé, dépassant la moyenne, d'où, un coefficient de variation supérieur à $100 \%$ dans tous les cas).

Le diagramme des quartiles de la distribution des CCI (Tableau 4) révèle que $75 \%$ des échantillons (Q3) ont des CCI < 195500 cell. /ml et 25\% des échantillons (Q1) ont des CCI < 32000 cell. $/ \mathrm{ml}$.

La valeur observée d'écart interquartile (Q3-Q1) est considérée bonne, puisqu'elle est légèrement inférieure à 200000 cell. /ml. La médiane (Q2) de cette distribution est de 73000 cell. /ml dont $50 \%$ des échantillons ont des CCI inférieurs à cette valeur médiane qui est relativement proche de la MG CCI (107500 cell. /ml) et largement éloignée de la MA CCI (371000 cell. /ml), ce qui témoigne l'utilité de l'utilisation de la MG dans la répartition des comptages cellulaires. En effet, la MA des CCI d'une lactation ne peut pas rendre compte de leur irrégularité qui caractérise également la présence d'infection, alors que la MG permet de ne pas surestimer l'importance d'un CCI élevé et apprécie de façon plus juste ce qui se passe dans la majorité de la population. Ce résultat conforme avec celui discerné par Sérieys [10], qui a utilisé la MG sur les données brutes (cell. $/ \mathrm{ml}$ ) et la transformation logarithmique pour le calcul des MA et des ET. La même évocation, concernant la grande différence entre la MA CCI et la médiane (Q2), a été signalée par M'Sadak et al. [15], lors de l'étude des quartiles des CCI des vaches laitières appartenant à des petits troupeaux bovins hors sol dans le Sahel Tunisien. L'étude a dévoilé, aussi, une valeur d'écart interquartile (Q3-Q1) élevée, et qui a été expliquée surtout par l'hétérogénéité marquée des CCI des échantillons considérés. 
Tableau 4. Répartition des quartiles des CCI

\begin{tabular}{lccc}
\hline \multirow{2}{*}{ Variables } & \multicolumn{3}{c}{ CCI (x1000 cell. /ml) } \\
\cline { 2 - 4 } & Ferme 1 & Ferme 2 & Echantillon total suivi \\
\hline Q1 & 32 & 32 & 32 \\
Q2 (Médiane) & 73 & 73 & 73 \\
Q3 & 207 & 193 & 195,5 \\
Q3-Q1 (Écart interquartile) & 175 & 161 & 163,5 \\
\hline
\end{tabular}

\section{2. Évolution cellulaire et productions laitières quantitative et qualitative relevées}

\subsubsection{Considérations générales}

Le CCI est une évaluation directe de la réponse immunitaire de la vache contre un agent infectieux de la glande mammaire. L'augmentation du CCI est accompagnée d'une réduction de la production laitière [13] et d'une réduction de la qualité du lait [24].

Les Figures 1 et 2 illustrent l'évolution du comptage cellulaire (MA CCI), de la production laitière (PL), des Matières Grasses (MG) et Protéiques (MP) et du taux d'urée, au cours de la période d'étude (Contrôles de C1 à C8) chez les 184 vaches considérées.

Il convient de noter, à ce niveau (Figure 1), que le contrôle laitier C1 n'a pas eu lieu au niveau de la ferme 1 .

\subsubsection{Relation entre comptage cellulaire et production laitière quantitative}

La MA CCI a évolué au cours de la lactation de façon inverse à la production laitière. Elle a été minimale au cours du dernier mois de contrôle (mois d'Avril), et maximale au quatrième contrôle. On remarque, ainsi, que l'élévation des CCI a un impact sur le niveau de production. Ce résultat a été vérifié par M'Sadak et al. [15, 25], lors d'un suivi des petits élevages bovins hors sol dans la région de Monastir et lors de l'étude de l'état sanitaire mammaire chez des élevages bovins hors sol de la région de Mahdia. Cependant, en examinant l'étude menée par Bousselmi et al. [2] sur plusieurs troupeaux de différentes régions de la Tunisie, on constate que l'effet du niveau de production sur le comptage cellulaire individuel n'a pas été significatif, dans les troupeaux appartenant au Nord comme dans le Sud Tunisien. 


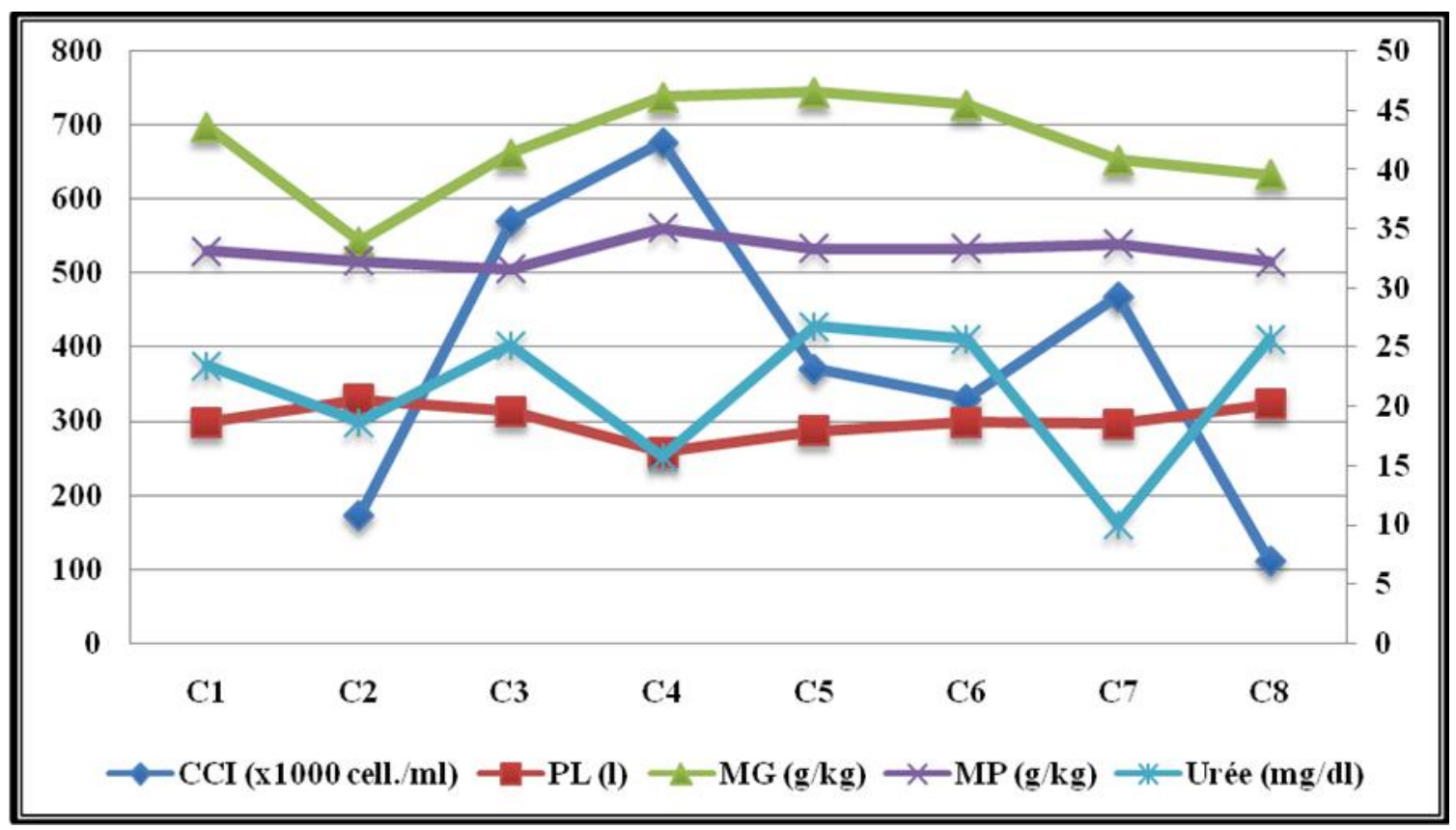

Fig. 1. Évolution du CCI moyen de la Ferme 1 au cours d'une lactation en relation avec les paramètres de production et de qualité laitières

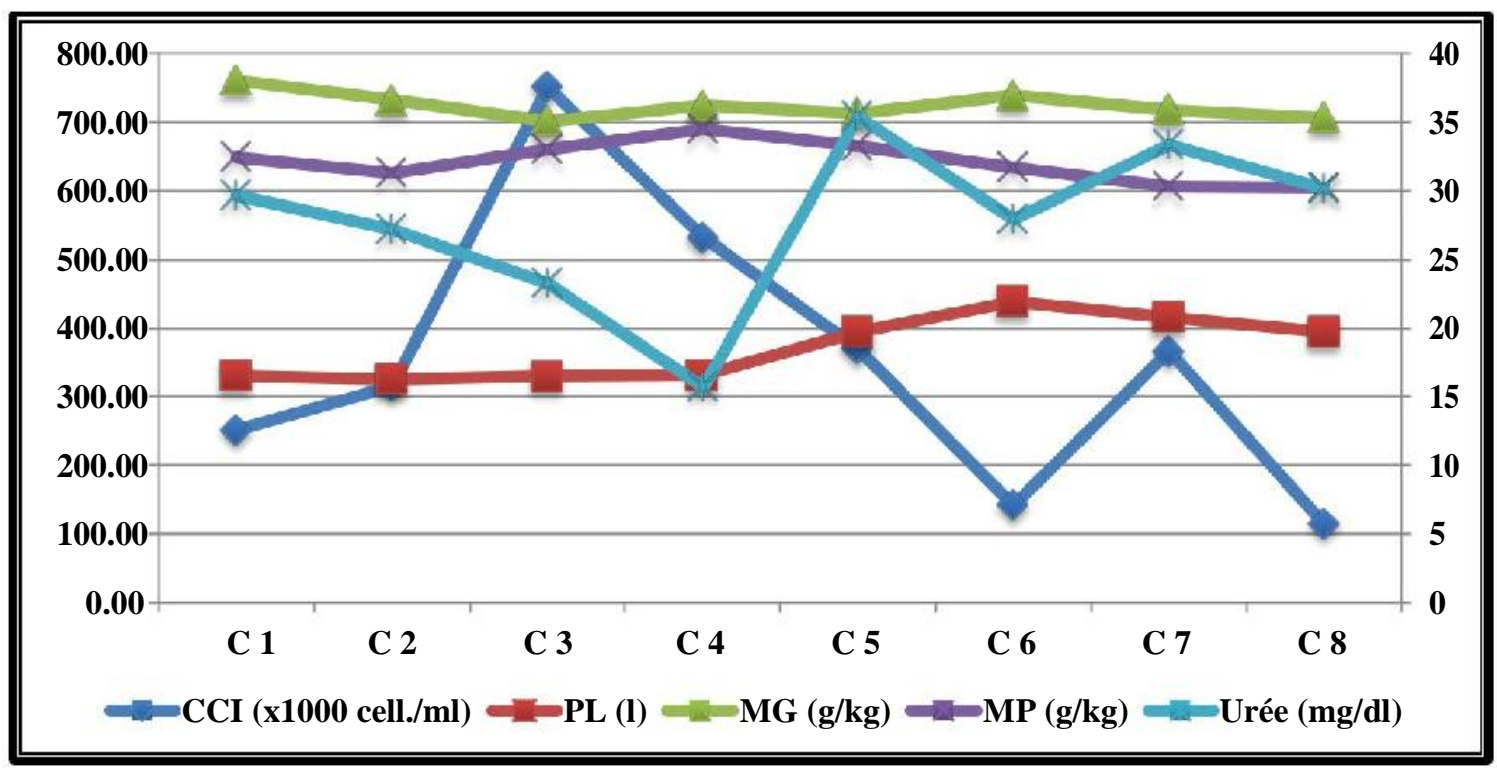

Fig.2. Évolution du CCI moyen de la Ferme 2 au cours d'une lactation en relation avec les paramètres de production et de qualité laitières

\subsubsection{Relation entre comptage cellulaire et production laitière qualitative}

L'étude entreprise a touché uniquement trois paramètres de qualité du lait, à savoir : $\mathrm{MG}(\mathrm{g} / \mathrm{kg})$, MP $(\mathrm{g} / \mathrm{kg})$ et Taux d'Urée $(\mathrm{mg} / \mathrm{dl})$. Au niveau national, le lait se caractérise par les valeurs moyennes suivantes : $34,7 \mathrm{~g} / \mathrm{kg} \pm 5,1$ pour la matière grasse et de $30,8 \mathrm{~g} / \mathrm{kg} \pm 3,1$ pour la matière 
protéique (APIA, 2008). Pour le taux d'urée, la plage de confort, est un taux situé entre 20 et 30 $\mathrm{mg} / \mathrm{dl}[26]$.

La corrélation entre le comptage des cellules somatiques individuelles et la composition du lait, notamment la matière protéique et la matière grasse, a été démontré hautement significative par Lindmark-Mansson et al. [27].

Cependant, d'après les Figures 1 et 2, les teneurs en matières grasses (MG) et protéiques $(\mathrm{MP})$ ont affiché des comportements différents de ceux que l'on avait prévues dans l'étude, en variant indépendamment des CCI ou de la PL. On peut expliquer la variation des taux de MG et MP, d'une façon différente dans les deux fermes, par l'intervention d'autres facteurs influant ces taux dans le lait, notamment la ration alimentaire, la saison, le stade de lactation, ... [28].

Les vaches ayant un taux d'urée faible dans le lait ont un risque de mammite accru [29]. La relation entre le taux d'urée, entre autres, la consommation du concentré, et le risque de mammite [30] est généralement inversée par rapport aux observations mises en œuvre. Cependant, cet impact est lié, aussi, au niveau de la production laitière [31], relation qui est par ailleurs considérée dans la présente étude. Une absence de relation entre mammite (Concentration cellulaire Somatique : CCS élevée) et le taux d'urée du lait au niveau élevage a été rapportée par Barnouin et Chassagne [32] et Godden et al. [33]. Mais, au niveau individuel, Godden et al. [33] ont dévoilé une augmentation de la CCS avec la diminution du taux d'urée du lait, ce qui rejoint les résultats obtenus. Ces auteurs évoquent la possibilité d'une sous-estimation de la teneur en urée du lait à forte CCS lors du dosage. Une autre hypothèse peut être avancée, c'est que les taux d'urée faibles peuvent provenir de problèmes de sous-consommation alimentaire [29].

\subsection{Répartition des CCI et des paramètres laitiers selon certains facteurs physiologiques et zootechniques}

\subsubsection{Distribution des CCI selon les facteurs de lactation}

Les deux Figures 3 et 4 confirment le résultat rapporté par Coulon et al. [11], Hanzen [34] et M'Sadak et al. [25] qui ont montré que les vaches primipares ont, en moyenne, des CCI faibles comparativement avec les vaches multipares. Ceci est dû particulièrement à un accroissement de l'effet de mammite avec l'âge. Il peut encore se créer à partir du résultat d'une réaction plus forte au niveau cellulaire contre une infection ou à une plus grande étendue des lésions permanentes de la mamelle se perpétrant comme résultante d'une infection chez les vaches âgées [35]. Pour cette raison, les élevages jeunes, où le pourcentage des vaches en 1ère et 2ème lactation est élevé, peuvent être relativement moins touchés par les élévations des taux cellulaires.

Pour l'effet du rang de lactation sur la quantité produite journalière, on a remarqué que, dans le troupeau de la ferme Gloulou, les primipares produisent moins de lait (18 1/j) que les multipares 
(25 l/j), alors qu'à la ferme OTD, il n'y a pas de différence entre les deux catégories. Pour les paramètres de qualité de lait (MG, MP et Urée), on n'a enregistré aucune différence significative entre les jeunes vaches et les vaches âgées, dans les deux élevages.

Le dépouillement des résultats CCI selon le rang et le stade de lactation des vaches, a confirmé les résultats trouvés par M'Sadak et al. [25,15].

Les travaux réalisés ont prouvé que les MA CCI et les MG CCI chez les primipares sont inférieures à celles des multipares. Mais, aussi, les MG CCI des primipares (75000cell. /ml) et les multipares (90000 cell. /ml) sont nettement moins élevées par rapport aux MA CCI des primipares ( 214000 cell. $/ \mathrm{ml}$ ) et des multipares (437000 cell. /ml). En plus du rang de lactation, le stade de lactation est un paramètre de variation des CCI.

En effet, dans les 100 premiers jours de lactation, à l'exception des jours de lactosérum, les MA CCI (74000 cell. /ml pour les primipares et 135000 cell. /ml pour les multipares) et les MG CCI (43000 cell. /ml pour les primipares et 59000 cell. $/ \mathrm{ml}$ pour les multipares) sont faibles. En contre partie, à la fin de la lactation, les CCI sont élevés (Tableau 5). De tels résultats concordent avec ceux relatés dans la bibliographie $[11,12,13,36,15]$.

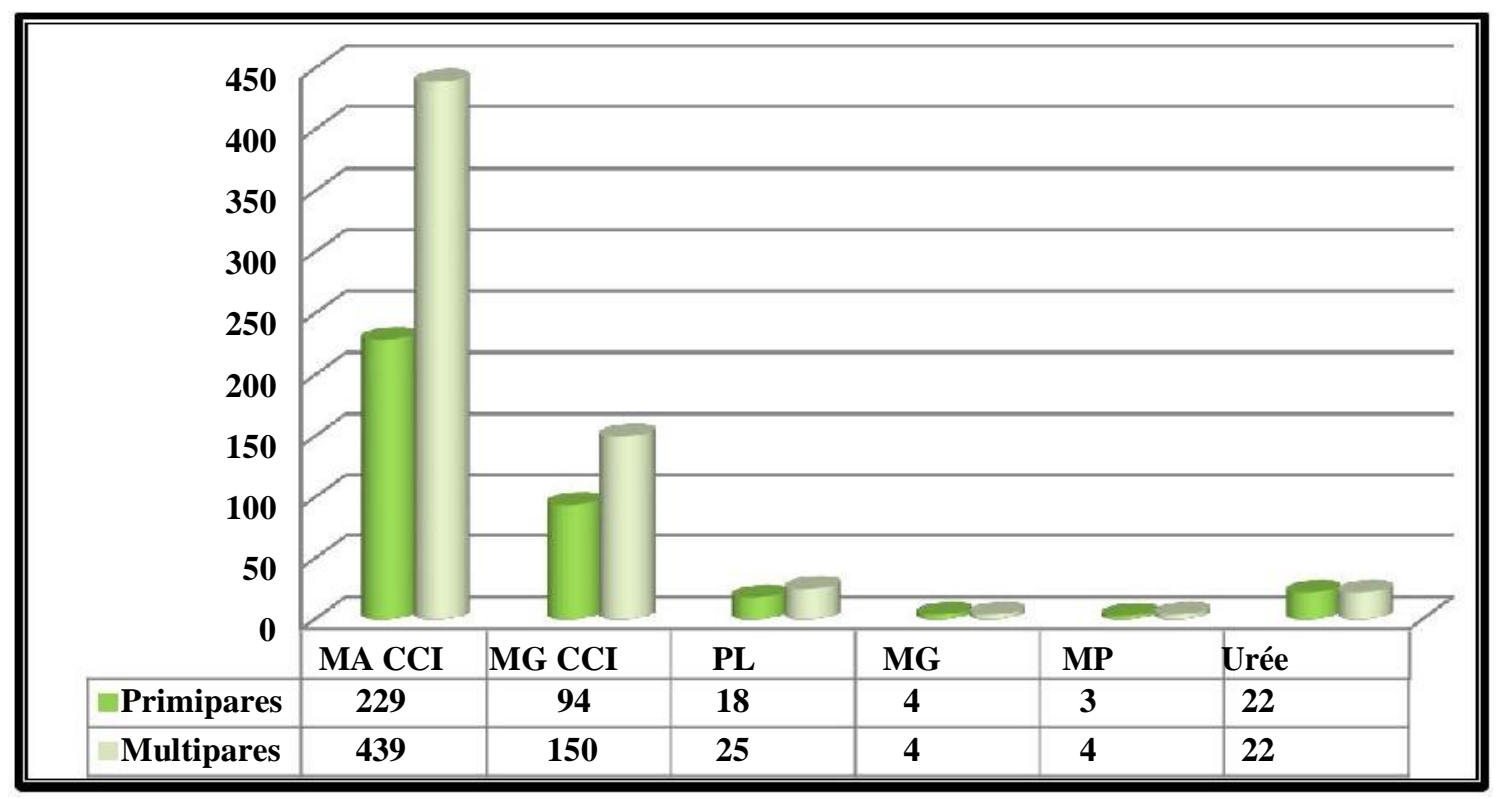

Fig.3. Répartition des vaches de la Ferme 1 d'après le rang de lactation 


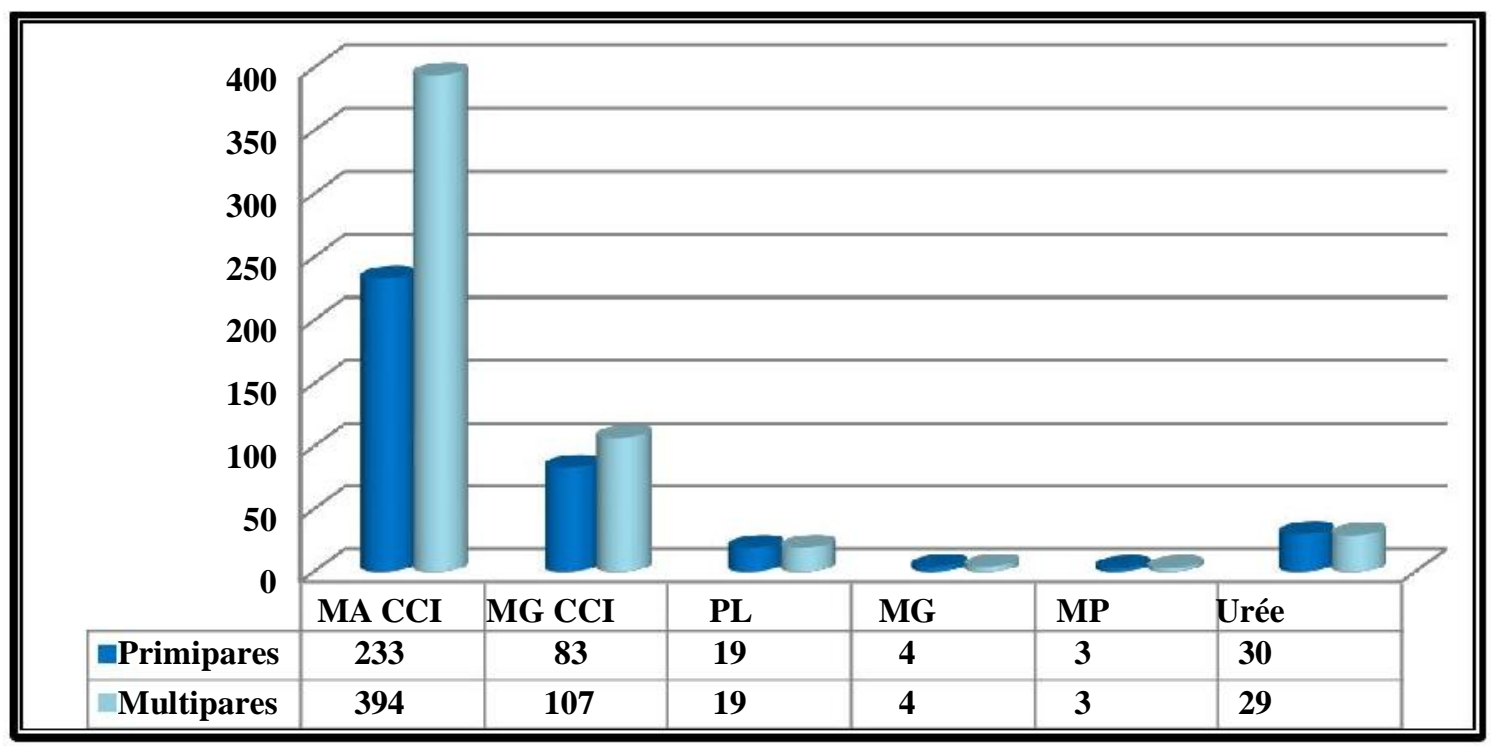

Fig.4. Répartition des vaches de la Ferme 2 selon le rang de lactation

Tableau 5. Variation des valeurs des CCI suivant le rang et le stade de lactation

\begin{tabular}{cccccccc}
\hline \multirow{2}{*}{ CCI (x } & \multirow{2}{*}{ 100 cell. /ml) } & \multicolumn{2}{c}{$<\mathbf{1 0 0} \mathbf{j}$} & \multicolumn{2}{c}{$\mathbf{1 0 0 - 2 0 0} \mathbf{j}$} & \multicolumn{2}{c}{$>\mathbf{2 0 0} \mathbf{j}$} \\
\cline { 3 - 8 } Ferme 1 & MA & MG & MA & MG & MA & MG \\
\cline { 3 - 8 } & Multipares & $59 \pm 57$ & 44 & $247 \pm 525$ & 97 & $274 \pm 286$ & 105 \\
\multirow{2}{*}{ Ferme 2 } & Primipares & $80 \pm 177$ & 73 & $408 \pm 1097$ & 103 & $1516 \pm 3518$ & 217 \\
& Multipares & $114 \pm 222$ & 52 & $382 \pm 2067$ & 82 & $877 \pm 3154$ & 179 \\
\hline \multirow{2}{*}{ Total } & Primipares & $74 \pm 87$ & 43 & $221 \pm 448$ & 82 & $457 \pm 886$ & 126 \\
& Multipares & $135 \pm 238$ & 59 & $391 \pm 1780$ & 89 & $1043 \pm 3250$ & 189 \\
\hline
\end{tabular}

\subsubsection{Répartition des CCI selon le mois de vêlage}

Le Tableau 6 montre la répartition des CCI des 180 vaches considérées des deux élevages suivis en fonction du mois de vêlage. On enregistre le plus faible CCI au mois d'Octobre (151000 cell./ml), alors que le CCI le plus élevé est enregistré au mois de Mars (923000 cell./ml). 
Tableau 6. Distribution des CCI (x 1000 cell. /ml) des vaches considérées des deux fermes suivant le mois de vêlage

\begin{tabular}{lcccccc}
\hline Mois de vêlage & Effectif & \% & Min. & Max. & MA CCI & MG CCI \\
\hline Janvier & 20 & 11 & 40 & 1257 & 395 & 135 \\
Février & 18 & 10 & 59 & 863 & 228 & 88 \\
Mars & 18 & 10 & 43 & 4675 & 923 & 193 \\
Avril & 12 & 7 & 39 & 540 & 218 & 84 \\
Mai & 2 & 1 & 138 & 583 & 361 & 121 \\
Juin & 7 & 4 & 110 & 828 & 389 & 132 \\
Juillet & 21 & 12 & 48 & 898 & 272 & 97 \\
Août & 12 & 7 & 24 & 2257 & 394 & 173 \\
Septembre & 18 & 10 & 29 & 953 & 265 & 105 \\
Octobre & 16 & 9 & 48 & 476 & 151 & 73 \\
Novembre & 17 & 9 & 44 & 459 & 221 & 88 \\
Décembre & 19 & 10 & 46 & 765 & 178 & 72 \\
\hline
\end{tabular}

\subsubsection{Distribution des CCI selon la saison de vêlage}

Le Tableau 7 montre la variation des CCI suivant la saison de vêlage. Les troupeaux suivis, ayant un schéma saisonnier de mises-bas, présentent une MA CCI plus élevée durant la période de vêlage de la saison printanière, et en second lieu, de la saison estivale. Les MA CCI les plus bas, ont été notées durant la saison automnale, et en second lieu, lors de la saison hivernale. Ces constatations diffèrent des résultats obtenus par M'Sadak et al. [15]. En effet, l'étude en question a été accomplie également dans une région du Sahel Tunisien (Mahdia), mais les saisons de vêlage qui ont influencé les comptages cellulaires ont été l'automne et l'hiver. La hausse des CCI durant les saisons printanière et estivale pourrait être expliquée, à la fois par une augmentation des nouveaux cas de CCI élevés au printemps et par la hausse des cas chroniques en été. Barkema \& Riekerink [37] ont expliqué cette élévation des CCI par l'augmentation des nouvelles infections qui pourrait être due à une plus grande incidence d'infections du pis causées par Staphylococcus aureus.

Tableau 7. Répartition des CCI (x 1000 cell. /ml) des vaches considérées des deux fermes selon la saison de vêlage

\begin{tabular}{lcccccc}
\hline Mois de vêlage & Effectif & \% & Min & Max & MA CCI & MG CCI \\
\hline Hiver & 57 & 32 & 40 & 1257 & 270 & 99 \\
Printemps & 32 & 18 & 39 & 4675 & 623 & 148 \\
Été & 40 & 22 & 24 & 2257 & 330 & 126 \\
Automne & 51 & 28 & 29 & 953 & 215 & 90 \\
\hline
\end{tabular}




\section{CONCLUSION}

Cette étude, accomplie dans la Tunisie littorale semi-aride chez deux grandes exploitations bovines laitières, a permis de dégager que $75 \%$ des vaches des deux troupeaux ont révélé une concentration cellulaire du lait inférieure à 200000 cell. /ml. En effet, la moyenne individuelle totale est de 371000 cell. /ml. On a enregistré, ainsi, des moyennes arithmétiques (MA) des CCI proches pour les deux élevages considérés, mais une grande hétérogénéité de la qualité cellulaire du lait produit, a été notée. Cette situation a engendré également des pourcentages faibles des vaches qui ne respectent pas le seuil de 200000 cell. /ml, considéré généralement comme seuil optimum, au moins à l'échelle européenne. La situation actuelle des deux élevages n'est pas tout à fait bienfaisante, car un taux de $20 \%$ des vaches infectées est à surveiller avec prudence, ce qui signifie que $20 \%$ des vaches dévoilent des pertes laitières. Par ailleurs, on peut dire que la moyenne géométrique (MG) est un paramètre plus fiable dans le calcul des moyennes des numérations cellulaires, méritant d'être valorisée dans la normalisation recherchée concernant l'élaboration d'un système de paiement du lait à la qualité à instaurer ensuite dans les Conditions Tunisiennes. Le diagramme des quartiles a été adopté pour mieux apprécier la répartition des données cellulaires au niveau de l'échantillon étudié. Il a confirmé l'utilité de l'utilisation de la MG au lieu de la MA dans l'analyse des moyennes cellulaires dans les deux élevages suivis du fait que les MG des CCI sont adjacentes des valeurs médianes des valeurs des CCI.

Dans l'ensemble, le CCI a affecté la production laitière quantitative d'une façon inverse. Aussi, on a remarqué que le CCI a dévoilé un accroissement avec le numéro de lactation, le stade de lactation, et la saison printanière de vêlage.

\section{RÉFÉRENCES}

[1] PROMET. Étude des déterminants de la qualité du lait. Rapport final. Société de Promotion et d'Études. Agence de Promotion des Investissements Agricoles (APIA), Ministère de l'Agriculture, Tunisie, 2008, $42 \mathrm{p}$.

[2] Bousselmi K., Djemali M., Bedhiaf S., Hamrouni A., Bedhiaf A. Facteurs de variation des Scores des Cellules Somatiques du lait de vache de race Holstein en Tunisie. Actes Renc. Rech. Ruminants, Paris, 2011, 18, p. 203.

[3] Noireterre P. Suivi de comptages cellulaires et d'examens bactériologiques lors de mammites cliniques chez la vache laitière. Thèse Vétérinaire, École Nationale Vétérinaire de Lyon, France, 2006, 98 p.

[4] Hoogeveen H., Huijps K., Lam T.J.G.M. Economic aspects of mastitis: new developments, New Zealand Veterinary Journal, vol. 59, 2011, 16-23. 
[5] Clerc P. Les mammites : Facteurs et indicateurs de risque, Chambre d'Agriculture des AlpesMaritimes, France, Avril 2016, 2 p.

[6] O'brien B, Meaney W, Mcdonagjh D., Kelly A. Influence of somatic cell count and storage interval on composition and processing characteristics of milk from cows in late lactation. Aust. Journal Dairy Tech., 56, 2001, 213-218.

[7] OSAM. Sélection animale et mammites : Situation actuelle et perspectives d'avenir, Wallonie Elevages, ${ }^{\circ} 2,2009,28-31$.

[8] Baillargeon J. Comptage de cellules somatiques : Un peu plus haut, un peu plus bas?, Flashmammite, vol. 1, $\mathrm{n}^{\circ}$ 2, 2004, 2 p.

[9] Remy D. Les mammites, Livre, France Agricole Éditions, 2010, 259 p.

[10] Sérieys F. Concentration cellulaire du lait individuel de vache : Influence de l'état d'infection mammaire, du numéro, du stade de lactation et de la production laitière. Ann. Rech. Vét. France, 16, 1985, 255-261.

[11] Coulon J.B., Dauver F., Garel J.P. Facteurs de variation de la numération cellulaire du lait chez des vaches laitières indemnes de mammites cliniques. INRA Prod. Anim. France, 1996, 9 (2) , 133-139.

[12] Barnouin J., Geromegnace N., Chassagne M., Dorr N., Sabatier P. Facteurs structurels de variation des niveaux de comptage cellulaire du lait et de fréquence des mammites cliniques dans 560 élevages bovins répartis dans 21 départements français. INRA Prod. Anim. France, 12, 1999, $39-48$.

[13] Rupp R., Boichard D., Bertrand C., Bazin S. Bilan national des numérations cellulaires dans le lait des différentes races bovines laitières françaises. INRA Prod. Anim. France, 2000, 13 (4), 257-267.

[14] Hanzen Ch. Propédeutique de la glande mammaire : Sémiologie et diagnostic individuel et de troupeau. Université de Liège, Belgique, 2009, 121 p.

[15] M'Sadak Y., Mighri L., Kraiem K. Étude des facteurs de variation des niveaux de comptage cellulaire individuel du lait chez des petits troupeaux bovins hors sol en Tunisie, Revue Nature \& Technologie, B- Sciences Agronomiques et Biologiques, Janvier 2013, nº 08, 48-52.

[16] Haj Mbarek R., M'Sadak Y. Facteurs de variation cellulaire du lait de vache chez des petits et moyens troupeaux hors sol menés en milieu semi-aride (Tunisie Littorale). Algerian Journal of Arid Environment (Algérie) vol.4, Juin 2014, n 1, 26-38.

[17] M'Sadak Y., Mighri L. Analyse descriptive et facteurs d'évolution des numérations cellulaires individuelles du lait chez des petits troupeaux bovins hors sol dans la région de Monastir (Tunisie), Algerian Journal of Arid Environment, vol. 5, Décembre 2015, n 2, 3-16. 
[18] M'Sadak Y., Haj Mbarek R., Mighri L. Facteurs d'évolution des comptages cellulaires du lait de vache en système hors sol (Tunisie semi-aride), Rev. Mar. Sci. Agron. Vét., vol. 4, 2016a, n 1 , 46-50.

[19] M'Sadak Y., Haj Mbarek R., Mighri L. Description and variation factors of individual cell counts of milk in of units bovins aboveground (Tunisian Sahel). J. Fundam. Appl. Sci., vol. 8, 2016b, $\mathrm{n}^{\circ} 1,61-72$.

[20] Goodger W.J., Farver T., Pelletier J., Johnson P., Desnayer G., Galland J. The association of milking management practices with bulk tank somatic cell counts. Prev. Vet. Med. USA, 1993, 15, 235-251.

[21] Fabre J.M., Bazin S., Faroult B., Cail P., Berthelot X. Lutte contre les mammites. Résultats d'enquête réalisée auprès de 1038 élevages français. Bulletin des GTV. France, 1996, (2), 13-16.

[22] Mtaallah B., Oubey Z., Hammami H. Estimation des pertes de production en lait et des facteurs de risque des mammites subcliniques à partir des numérations cellulaires de lait de tank en élevage bovin laitier. Rev. Méd. Vét., 2002, 153, 4, 251-260.

[23] Mighri L., M’Sadak Y., Kraiem K.Analyse des numérations cellulaires du lait en élevage bovin hors sol dans la région de Monastir. Actes Renc. Rech. Ruminants, Paris, 2011, 18, p. 274.

[24] Durocher J., Perreault J.Y. Un comptage des cellules somatiques : Un outil indispensable pour gérer la santé du pis. Le Producteur de Lait Québécois. Canada, Novembre 2009, 28-30.

[25] M'Sadak Y., Mighri L., Ben Omrane H., Kraiem K. Étude de la situation sanitaire mammaire et estimation des pertes laitières chez des élevages bovins hors sol dans la région de Mahdia (Tunisie), Revue des BioRessources, 2 (2), 2012, 17-28.

[26] Perrussel E. Taux d'urée du lait : Un repère pour l'alimentation des troupeaux laitiers, élevage, l'information agricole du Rhône, $\mathrm{n}^{\circ}$ 2018, 18 juin 2009, 1 p.

[27] Lindmark-Mansson H., Branning C., Alden G., Paulsson, M. Relation entre le nombre de cellules somatiques, les populations de leucocytes individuels et les composants du lait en quart de lait de la mamelle bovine. International Dairy Journal, 2006, 16, 717-727.

[28] Snowdon M. Composition et valeur nutritive du lait, Ministère de l'Agriculture, l'Aquaculture et des Pêches, Nouveau Brunswick Canada, 1992, 92-2.

[29] Bareille, N., Beaudeau, F., Billon, S., Robert, A., Faverdin, P. Livest. Prod. Sci., 2003, 83, 53-62.

[30] Fraser D, Leaver J. A system study of high and low concentrate inputs for dairy cows: milk yield and composition, liveweight, feed intake, fertility and health over 4 years. Res Dev Agric 5, 1988, 93-101. 
[31] Pryce J., Brotherstone S. Estimation of lifespan breeding values in the UK and their relationship with health and fertility traits, Interbull, Bulletin, 1999, 21, 166-169.

[32] Barnouin J., Chassagne M. Les facteurs associés à l'incidence de la mammite clinique dans les troupeaux laitiers français au cours de la fin de la gestation et l'allaitement précoce. Vet. Res., 1998, 29, 159-171.

[33] Godden S.M. , Lissemore K.D, Kelton D.F. et al. Validation analytique d'un dosage de l'urée du lait infrarouge et effets des facteurs d'acquisition d'échantillons sur les résultats de l'urée du lait. J. Dairy Sci., v.83, 2000, 435-442.

[34] Hanzen Ch. Propédeutique de la glande mammaire : Sémiologie et diagnostic individuel et de troupeau. Université de Liège, Belgique, 2009, R21, 28 p.

[35] Leslie K.E. Somatic Cell Counts: Interpretation for Individual Cows. FACTSHEET, Ministère de l'Agriculture et de l'Alimentation Ontario, 2012, 03/85, Commande № 24-012.

[36] Hanzen Ch. Physio-anatomie et propédeutique de la glande mammaire. Université de Liège, Belgique, 2013, 170 p.

[37] Barkema H., Riekerink R.O. Effet des saisons sur le comptage de cellules somatiques et l'incidence de la mammite clinique, Cultiver les connaissances pour du lait de qualité. Le Réseau Canadien de Recherche sur la Mammite Bovine (RCRMB), Canada, 2008, 10-11.

\section{How to cite this article:}

M'Sadak Y. and Hamed I. Dairy production and sources of cellular variability of milk of cow's in two large farms in semi-arid environment (Tunisie coastal). J. Fundam. Appl. Sci., 2017, 9 (2), 861-877. 